\title{
Search for solar neutrons during the maximum activity of solar cycle 24
}

\author{
Diego Lopez; Yutaka Matsubara ${ }^{\dagger}$, Yasushi Muraki, Takashi Sako \\ Solar-Terrestrial Environment Laboratory, Nagoya University \\ 1 Furo-cho, Chikusa-ku, Nagoya 464-8601, Japan \\ E-mail: ndlopez@stelab.nagoya-u.ac.jp, ymatsu@stelab.nagoya-u.ac.jp
}

\begin{abstract}
During the second period of maximum solar activity (October 2013 through October 2014) of the current solar cycle we have searched for solar neutron events. When a solar flare occurs, ions are sometimes accelerated. Those ions interact with the solar atmosphere and produce solar neutrons. We examined recent data from six stations of the International Network of Solar Neutron Telescopes (Gornergrat, Switzerland; Mauna Kea, USA; Mt. Chacaltaya, Bolivia; Mt. Norikura, Japan; Mt. Sierra Negra, Mexico and Yangbajing, China). We report that no statistically significant solar neutron event associated to a solar flare has been found. Therefore, we calculated the upper limit of the neutron fluxes at the Sun based on a statistical analysis and considering impulsive emission. We report that our upper limits are consistent with the successful detections of solar neutrons made in solar cycle 23.
\end{abstract}

The 34th International Cosmic Ray Conference,

30 July- 6 August, 2015

The Hague, The Netherlands

\footnotetext{
*Corresponding author

${ }^{\dagger}$ Speaker.
} 


\section{Introduction}

Solar flares are large explosions that occur in the solar surface. They can sometimes accelerate ions to very high energies. The interaction of these ions with the solar atmosphere can produce via nuclear interactions neutrons among other particles. Understanding the mechanism of acceleration in a solar flare is of primary importance when studying solar neutrons. Solar neutrons can arrive straightforward from the Sun to the Earth without being affected by the interplanetary magnetic fields. Hence, they will carry key information concerning the original particle acceleration.

Since solar neutrons are not the only particle which are accelerated in a solar flare, we can also observe $\gamma$-rays, X-rays, protons, electrons and more. Massless particles will arrive at Earth faster than neutrons, thus we can consider these massless neutral particles as an indicator which shows solar neutrons are produced. In particular X-rays are a common indicator of the intensity of solar flares, and may be used as a sign of solar neutrons. X-rays are mainly observed by satellites, one of these is the Geostationary Operational Environmental Satellite (GOES-15), which records

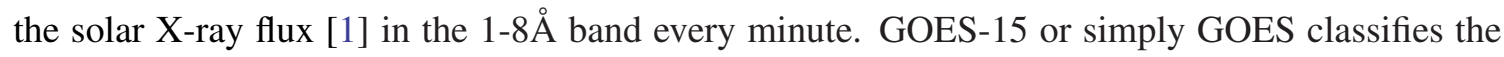
$\mathrm{X}$-ray flux intensity according to the flare size. This classification has five classes, A, B, C, M and $\mathrm{X}$. Assuming that neutrons are produced instantaneously as X-rays, they will reach the Earth and our detectors delayed from X-rays. The arrival time of neutrons depends on their energies because neutrons have mass. Neutrons with energies of $1 \mathrm{GeV}$ and $100 \mathrm{MeV}$ require about $1.2 \mathrm{~min}$ and 11 min longer than X-rays' travel time to reach the top of the Earth's atmosphere.

In the past solar cycle a number of six solar neutron events associated with large ( $>$ X1.0-class) solar flares have been reported [2,3]. Up to date, the number of positive solar neutron detections is twelve. Such small number of positive detections motivate us to continue with our search for more solar neutron events during the current solar cycle. To do so, we focused our analysis on the period of maximum activity, i.e. October 2013 to October 2014 where $21 \mathrm{M}$-class and $20 \mathrm{X}$-class flares occurred. A similar analysis covering a wider period is in progress and will be reported in a separate paper.

\section{The solar neutron detector}

Solar neutron monitoring for a long time has been carried out by Neutron Monitors (NM) which were originally designed to continuously measure the cosmic ray intensity. Actually, the first ground-based detection of solar neutrons was made by the NM [4]. However, NMs cannot measure the neutron's energy, because such information is lost in the producer. Therefore, Muraki et al., (1992) [5] proposed the Solar neutron telescope (SNT) which basically is a scintillator detector. It can discriminate between incident charged and neutral particles, measure the energy, and determine the direction of the recoiled protons. Its capability was demonstrated when clear solar neutron signals were observed during solar cycle 22 by the $1-\mathrm{m}^{2} \mathrm{SNT}^{1}$ installed in Norikura on 1991 June 4 [5] and during solar cycle 23 by the SNTs installed in Mt. Chacaltaya, Bolivia and Mt. Sierra Negra, Mexico on 2005 September 7 [3]. Since 1991 a number of seven SNTs have been installed around the world to conform the International Network of Solar Neutron Telescopes. In Table 1 the geographical location and the start date of the operation of the SNTs are shown. These detectors

\footnotetext{
${ }^{1}$ Nowadays this small-size SNT is no longer in operation.
} 
Table 1: International Network of Solar Neutron Telescopes

\begin{tabular}{llrrrccc}
\hline Station & Location & Long & Lat & $\begin{array}{r}\text { Height } \\
{[\mathrm{m}]}\end{array}$ & $\begin{array}{c}\text { Air mass } \\
{\left[\mathrm{g} / \mathrm{cm}^{2}\right]}\end{array}$ & $\begin{array}{c}\text { Area } \\
{\left[\mathrm{m}^{2}\right]}\end{array}$ & Operation \\
\hline ARAM & Aragats, Armenia & $40.5^{\circ} \mathrm{E}$ & $44.2^{\circ} \mathrm{N}$ & 3200 & 670 & 4 & 2003 Feb $\sim$ \\
GOSW & Gornergrat, Switzerland & $7.8^{\circ} \mathrm{E}$ & $46.0^{\circ} \mathrm{N}$ & 3135 & 690 & 4 & $1998 \mathrm{Jan} \sim$ \\
MKUS & Mauna Kea, USA & $156.3^{\circ} \mathrm{W}$ & $19.8^{\circ} \mathrm{N}$ & 4200 & 610 & 8 & $1997 \mathrm{Apr} \sim$ \\
$\mathrm{CHBO}$ & Mt. Chacaltaya, Bolivia & $68^{\circ} \mathrm{W}$ & $16.2^{\circ} \mathrm{S}$ & 5250 & 540 & 4 & $1992 \mathrm{Sep} \sim$ \\
$\mathrm{SNMX}$ & Mt. Sierra Negra, Mexico & $97.3^{\circ} \mathrm{W}$ & $19.0^{\circ} \mathrm{N}$ & 4580 & 575 & 4 & 2003 Jun $\sim$ \\
NRJP & Mt. Norikura, Japan & $137.5^{\circ} \mathrm{E}$ & $36.1^{\circ} \mathrm{N}$ & 2770 & 730 & 64 & $1996 \mathrm{Oct} \sim$ \\
YACH & Yangbajing, China & $90.5^{\circ} \mathrm{E}$ & $30.0^{\circ} \mathrm{N}$ & 4300 & 600 & 9 & $1999 \mathrm{Sep} \sim$ \\
\hline
\end{tabular}

are installed at low-latitude and high-altitude locations. With these conditions we can monitor solar neutrons as long period as possible. The high altitude is required to reduce the neutron's absorption in the Earth's atmosphere. Details of each SNT were described in $[6,7,8]$ and the references therein. In this paper we briefly explain the SNT components and the measurement method. To begin, all SNTs have a target layer which consists of a thick scintillator (20 to 50 $\mathrm{cm}$ ). Photomultipliers tubes (PMTs) are set on top or side of the target scintillators. Proportional counters (PRCs) operate as veto-counters and surround the detector. Underneath the target of some SNTs an array of PRCs is set to determine the arrival direction.

The detection process in the target is as follows. Incoming neutrons are converted into protons by nuclear interactions. Resulting recoil protons which are subjected to a charge exchange process tend to be scattered in the direction of incident neutrons, almost conserving their energy. The ionization energy loss of the protons is measured by PMTs. The discriminator thresholds of the PMTs are set at different energy levels. NRJP is set at $>20 \mathrm{MeV},>40 \mathrm{MeV},>80 \mathrm{MeV},>120 \mathrm{MeV}$ and $>160 \mathrm{MeV}$. ARAM, GOSW and YACH are set at $>40 \mathrm{MeV},>80 \mathrm{MeV},>120 \mathrm{MeV}$, and $>160 \mathrm{MeV}$. CHBO is set at $>40 \mathrm{MeV},>80 \mathrm{MeV},>160 \mathrm{MeV}$, and $>240 \mathrm{MeV}$. MKUS is set at $>12 \mathrm{MeV}$ (PMT-L) and $>20 \mathrm{MeV}$ (PMT-H). Finally, SNMX is set at $>30 \mathrm{MeV},>60 \mathrm{MeV}$, $>90 \mathrm{MeV}$, and $>120 \mathrm{MeV}$. The particle discrimination is done in the following way. When an incident particle hits the veto-counters, an electrical signal is produced. Now, if that signal is in electronical coincidence with the signal produced in the target, they will be recognized as charged particles. We call this operation mode "without-anti". The anti-coincidence of the veto-counter signals is the operational mode called "with-anti", in this mode the signals are identified as neutral particles.

The performance of the SNTs has been examined by different authors. In the present work we will use their results. The detection efficiencies of the SNTs installed in Norikura (NRJP) and Chacaltaya (CHBO) were performed via an accelerator experiment and also via a MonteCarlo simulation done by Tsuchiya et al.[6]. The detection efficiency of GOSW was performed by M. Moser [9] via MonteCarlo simulations using Geant3. K. Watanabe [7] performed a similar calculation (by using Geant4) to the detection efficiencies of MKUS and YACH. The detection efficiency of SNMX was calculated by L.X. González et al., [10] also using Geant4. In this work, we adopted the NM response calculated by Clem \& Dorman [11]. 


\section{Data Sample}

We focused our search for solar neutrons during the period of maximum solar activity which corresponds from October 2013 to October 2014. In this period, more than 41 solar flares classified as greater than M5 were recorded. Observation on the ground was carried by the SNTs and the Chacaltaya NM (CHNM). Due to the location of each SNT we have some limitations we need to consider. Our conditions to analyze events are that at the peak time the zenith angle of and the line-of-sight air mass to the Sun must be less than $65^{\circ}$ and $1000 \mathrm{~g} / \mathrm{cm}^{2}$ respectively. With these considerations, our final flare sample consists of 29 solar flares which we list in Table 2.

\section{Analysis}

We consider as an indicator of solar neutron production the time of maximum intensity of the SXR flux ( $t_{p}$ peak time in UT, $t_{0}$ in local time) recorded by GOES. We constructed 3-min count-rate profiles for each of the flares listed in Table 2. Then we set two windows, the work window and the signal window, to search for solar neutrons. The work window runs from $t_{0} \pm 3 \mathrm{~h}$ and the signal window runs from $t_{0}-15 \mathrm{~min}<t<t_{0}+45 \mathrm{~min}$. The first interval includes the backgrounds and the second interval the possible excess due to solar neutrons. To estimate the background we calculated the running average for 20 min excluding the signal window. In the signal window we interpolate data instead. In Figure 1 we show four examples of the studied events for four SNTs. In these plots the whole interval corresponds to the work window. The signal window is here enclosed by two vertical lines and a double-direction violet arrow. In Figure 1 we show only the lowest threshold of four SNTs. However, in our analysis we used the four different threshold levels from the with-anti operation mode of six SNTs and searched for solar neutrons.

\section{Results}

\subsection{Significance Distributions}

Having constructed the time profiles and defined the work windows for each flare, we determined the statistical significance of each profile. We proceeded in the following way. First, we calculated the deviation of each 3-min count from the average background excluding the signal window and normalized by standard deviation. Second, we built a background distribution with these data. Then, we fitted the resulting distribution to a Gaussian function. We built another distribution with the data from the signal window, and in the case we see an excess of events considerably significant equal or greater than $3 \sigma$, we can regard it as a candidate for a solar neutron event associated with the solar flare.

In Figure 2 we show as an example of our analysis the statistical significances for one of the largest flares in our list, the X4.9 flare which occurred on 2014 February 25. At the maximum intensity time of SXRs the Mauna Kea SNT (MKUS) was in good position to observe solar neutrons $\left(\theta_{\odot}=44^{\circ}\right.$, air mass $\left.=733 \mathrm{~g} / \mathrm{cm}^{2}\right)$. We can see isolated events which surpassed the $3 \sigma$ level in three channels of MKUS, but they are not consistent with respect to the other channels. 
Table 2: List of Soft X-ray flares observed by GOES (1-8̊̊) and the SNTs local time of possible observation.

\begin{tabular}{|c|c|c|c|c|c|c|c|c|c|}
\hline \multirow[b]{2}{*}{ Date } & \multicolumn{5}{|c|}{ Solar Flare } & \multicolumn{4}{|c|}{ Observation Site } \\
\hline & $\begin{array}{l}\text { Start } \\
(\mathrm{UT})\end{array}$ & $\begin{array}{c}t_{P}{ }^{a} \\
\text { (UT) }\end{array}$ & Class & Position & Region & $\mathrm{SNT}^{b}$ & $\begin{array}{c}t_{0}^{c} \\
(\mathrm{LT})\end{array}$ & $\begin{array}{c}\theta_{\odot}^{d} \\
{[\mathrm{deg}]}\end{array}$ & $\begin{array}{c}\text { Air Mass } \\
\\
{\left[\mathrm{g} / \mathrm{cm}^{2}\right]}\end{array}$ \\
\hline 2013 Oct 24 & $00: 21$ & $00: 30$ & M9.3 & S10E08 & 1877 & MKUS & $14: 30$ & 47 & 766 \\
\hline 2013 Oct 25 & $07: 53$ & 08:01 & $\mathrm{X} 1.7$ & S08E73 & 1882 & YACH & 14:01 & 54 & 830 \\
\hline \multirow[t]{2}{*}{2013 Oct 25} & $14: 51$ & $15: 03$ & $\mathrm{X} 2.1$ & S06E69 & 1882 & SNMX & $10: 03$ & 56 & 831 \\
\hline & & & & & & $\mathrm{CHBO}$ & $11: 03$ & 18 & 546 \\
\hline 2013 Oct 28 & $04: 32$ & 04:41 & M5.1 & N08W71 & 1875 & YACH & $10: 41$ & 46 & 737 \\
\hline 2013 Oct 29 & $21: 42$ & $21: 54$ & $\mathrm{X} 2.3$ & N08W71 & 1875 & MKUS & $11: 54$ & 34 & 665 \\
\hline \multirow[t]{2}{*}{2013 Nov 01} & $19: 46$ & $19: 53$ & M6.3 & S11E03 & 1884 & SNMX & 1353 & 42 & 678 \\
\hline & & & & & & $\mathrm{CHBO}$ & $15: 53$ & 52 & 733 \\
\hline 2013 Nov 05 & $22: 07$ & $22: 12$ & $\mathrm{X} 3.3$ & S12E46 & 1890 & MKUS & $12: 12$ & 36 & 676 \\
\hline 2013 Dec 31 & $21: 45$ & $21: 58$ & M6.4 & S16W35 & 1936 & MKUS & $11: 58$ & 43 & 729 \\
\hline \multirow[t]{2}{*}{2014 Jan 1} & $18: 40$ & $18: 52$ & M9.9 & S14W47 & 1936 & SNMX & $12: 52$ & 42 & 681 \\
\hline & & & & & & $\mathrm{CHBO}$ & $14: 52$ & 33 & 587 \\
\hline \multirow[t]{2}{*}{2014 Jan 07} & $18: 04$ & $18: 32$ & $\mathrm{X} 1.2$ & S15W11 & 1944 & SNMX & $12: 32$ & 41 & 674 \\
\hline & & & & & & $\mathrm{CHBO}$ & $14: 32$ & 27 & 567 \\
\hline 2014 Jan 30 & $15: 48$ & $16: 11$ & M6.6 & S13E58 & 1967 & $\mathrm{CHBO}$ & $12: 11$ & 8 & 540 \\
\hline 2014 Feb 25 & $00: 39$ & $00: 49$ & $\mathrm{X} 4.9$ & S12E82 & 1990 & MKUS & $14: 49$ & 44 & 733 \\
\hline 2014 Mar 12 & $22: 28$ & $22: 34$ & M9.3 & N15E78 & 1996 & MKUS & $12: 34$ & 23 & 625 \\
\hline \multirow[t]{2}{*}{2014 Mar 29} & $17: 35$ & $17: 48$ & $\mathrm{X} 1.0$ & N11W32 & 2017 & SNMX & $11: 48$ & 19 & 582 \\
\hline & & & & & & $\mathrm{CHBO}$ & $13: 48$ & 26 & 564 \\
\hline 2014 Apr 2 & $13: 18$ & $14: 05$ & M6.5 & N14E53 & 2027 & GOSW & $16: 05$ & 53 & 925 \\
\hline 2014 Apr 18 & $12: 31$ & 13:03 & M7.3 & S20W34 & 2036 & GOSW & $15: 03$ & 40 & 794 \\
\hline 2014 Apr 25 & $00: 17$ & $00: 27$ & $\mathrm{X} 1.3$ & S14W89 & 2035 & MKUS & $14: 27$ & 31 & 653 \\
\hline 2014 May 08 & 09:59 & 10:07 & M5.2 & N08E54 & 2056 & GOSW & 12:07 & 33 & 746 \\
\hline 2014 Jun 10 & $11: 36$ & $11: 42$ & $\mathrm{X} 2.2$ & S19E81 & 2087 & GOSW & $13: 42$ & 23 & 706 \\
\hline 2014 Jun 10 & $12: 36$ & $12: 52$ & $\mathrm{X} 1.5$ & S20E89 & 2087 & GOSW & $14: 52$ & 29 & 724 \\
\hline 2014 Jun 11 & $08: 59$ & 09:06 & $\mathrm{X} 1.0$ & S18E66 & 2087 & GOSW & 11:06 & 37 & 767 \\
\hline 2014 Aug 24 & $12: 00$ & $12: 17$ & M5.9 & S07E75 & 2151 & GOSW & $14: 17$ & 36 & 764 \\
\hline \multirow[t]{2}{*}{2014 Sep 10} & $17: 21$ & $17: 45$ & $\mathrm{X} 1.6$ & N14E02 & 2158 & SNMX & $12: 45$ & 17 & 580 \\
\hline & & & & & & $\mathrm{CHBO}$ & $13: 45$ & 28 & 570 \\
\hline \multirow[t]{2}{*}{2014 Oct 02} & $18: 49$ & 19:01 & M7.3 & S17W82 & 2192 & SNMX & 14:01 & 25 & 596 \\
\hline & & & & & & $\mathrm{CHBO}$ & 15:01 & 41 & 633 \\
\hline 2014 Oct 22 & $14: 02$ & $14: 28$ & $\mathrm{X} 1.6$ & S14E13 & 2192 & $\mathrm{CHBO}$ & $10: 28$ & 27 & 566 \\
\hline 2014 Oct 24 & $21: 07$ & $21: 41$ & $\mathrm{X} 3.1$ & S16W21 & 2192 & MKUS & $11: 41$ & 32 & 659 \\
\hline 2014 Oct 25 & $16: 55$ & $17: 08$ & $\mathrm{X} 1.0$ & S16W31 & 2192 & SNMX & $12: 08$ & 35 & 635 \\
\hline 2014 Oct 27 & 00:06 & $00: 34$ & M7.1 & S14W44 & 2192 & MKUS & $14: 34$ & 49 & 781 \\
\hline \multirow[t]{2}{*}{2014 Oct 27} & $14: 12$ & $14: 47$ & $\mathrm{X} 2.0$ & S17W52 & 2192 & SNMX & 08:47 & 60 & 891 \\
\hline & & & & & & $\mathrm{CHBO}$ & $10: 47$ & 22 & 553 \\
\hline
\end{tabular}

${ }^{a}$ GOES SXR maximum peak time. ${ }^{b}$ Observatory where neutrons have greater possibilities.

${ }^{c}$ Peak time in local time. ${ }^{d}$ Solar zenith angle at the peak time. ${ }^{e}$ Line-of-sight air mass at the peak time. 

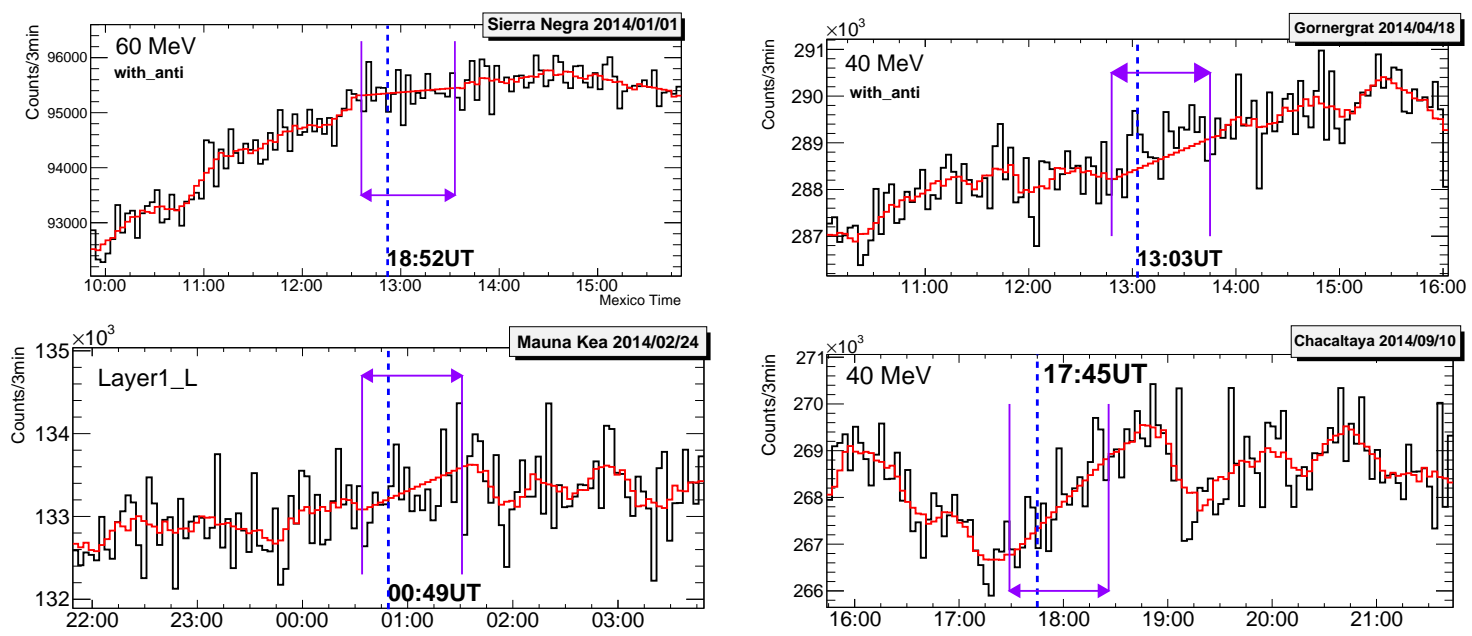

Figure 1: The 3-min count-rate profiles of four SNTs for two M-class and two X-class flares. The topleft and -right panels indicate those for the M9.9 (2014/01/01) and M7.3 (2014/04/18) flares respectively. The bottom-left and -right panels indicate those for the X4.9 (2014/02/25) and X1.6 (2014/09/10) flares respectively. The interval of the signal window is indicated by two horizontal and one vertical violet lines. The red thick line represents the 20-min average background. The dashed blue line and the label time show the flare peak time $\left(\mathrm{t}_{p}\right)$ according to GOES. The abscissa in all plots represents the time in UT unless indicated.
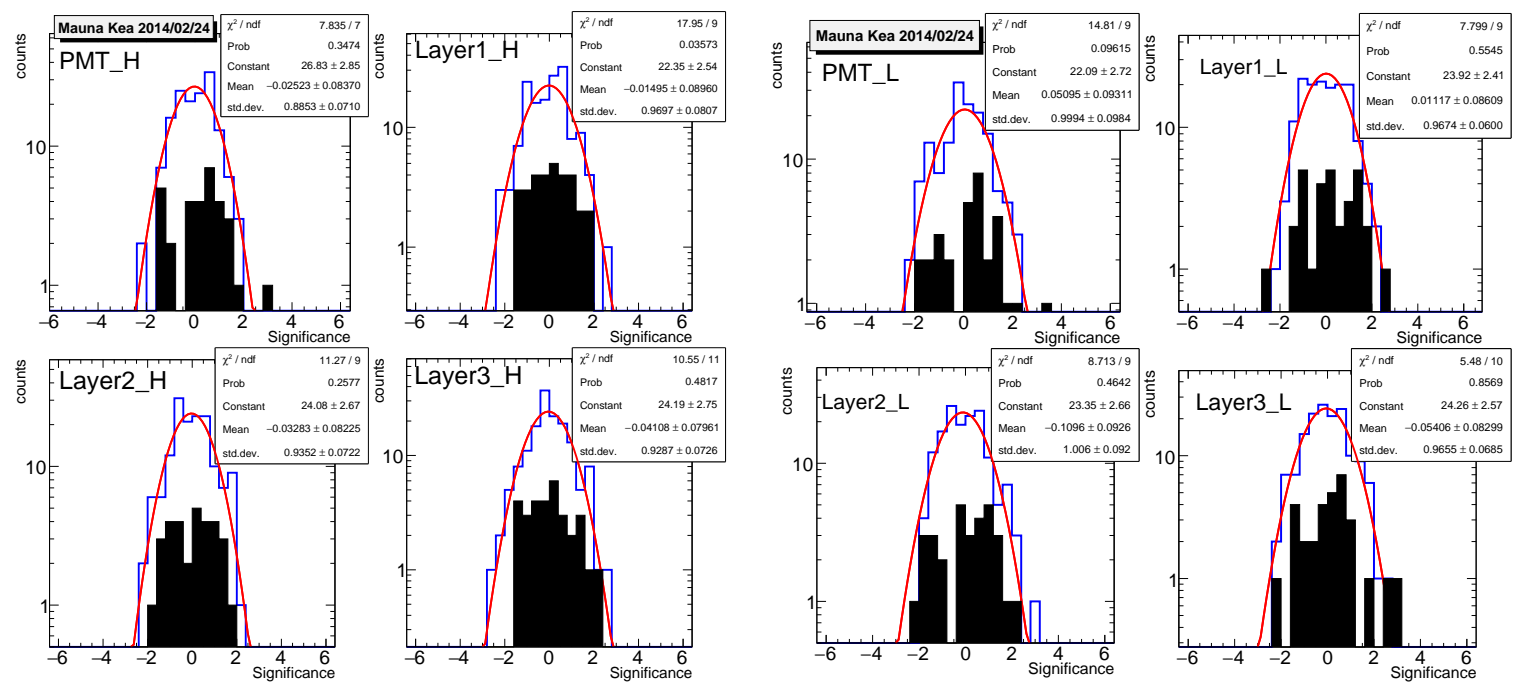

Figure 2: Statistical significances for eight different channels of MKUS for the largest flare in our list, the X4.9 (2014/02/25) flare. In all plots the overlapped black-shadowed area corresponds to the signal distribution. In the first top set of four, the results for the PMT-H with its corresponding 3 layers. In the second set, the distributions for the PMT-L also with its corresponding 3 layers. 

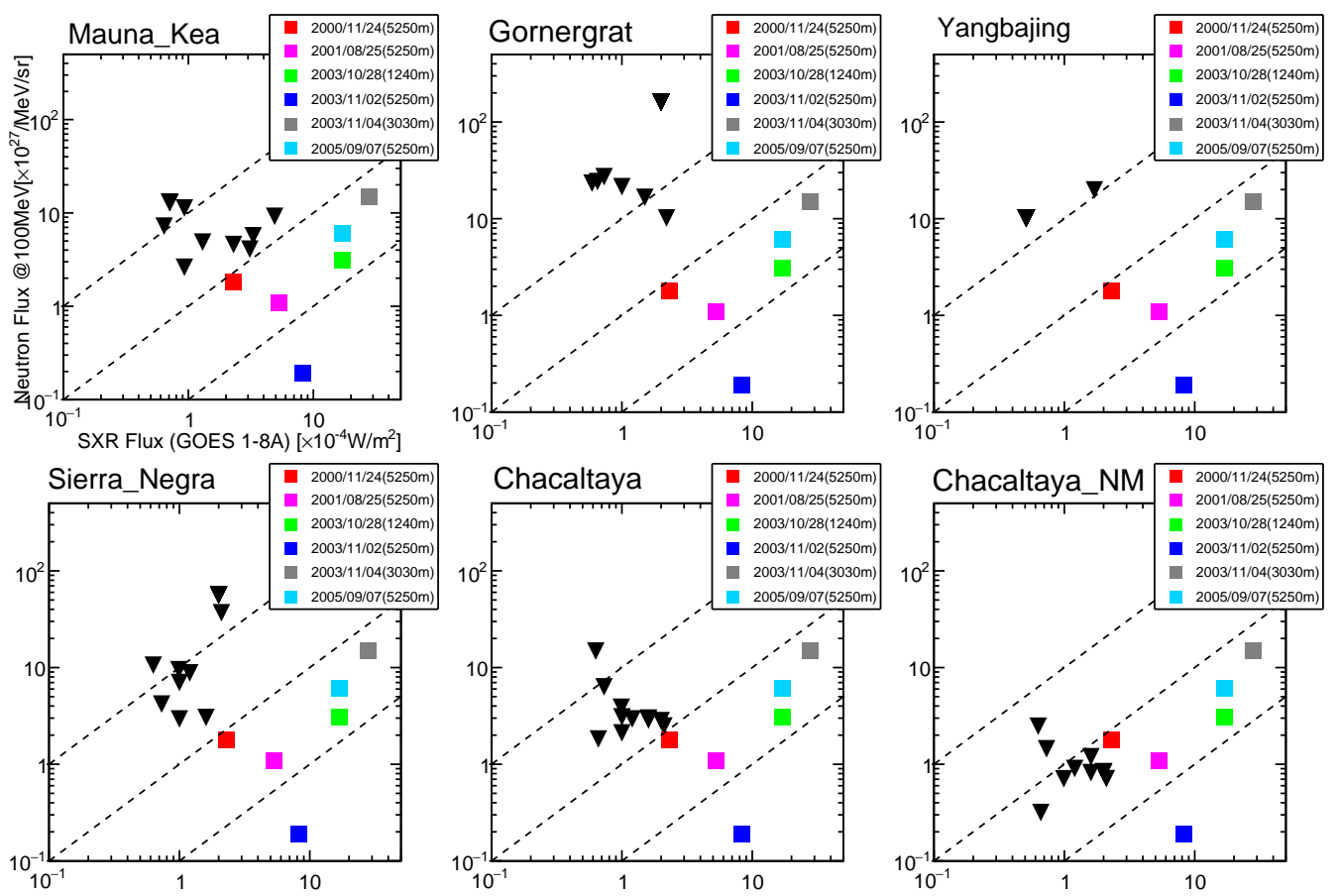

Figure 3: The $90 \%$ CL upper limits at $100 \mathrm{MeV}$ solar neutron flux at the Sun (ordinate) estimated for the lowest threshold level of five SNTs and one NM are plotted (black inverted triangles). Previous successful detections observed in cycle 23 [2, 3] are also plotted (colored squares). The abscissa represents the SXR flux (flare class) measured by GOES in the $1-8 \AA$ band.

\subsection{Estimation of the Upper Limit}

We performed the statistical analysis described in the previous section for all the events listed in Table 2. However, we could not find any statistically significant result. Nonetheless, we can calculate the upper limit of solar neutrons. To perform such estimation we considered the lowest threshold level of each detector. Although $>30 \mathrm{MeV}$ is the lowest energy threshold level of SNMX, we preferred to use the following level which is $>60 \mathrm{MeV}$. We did this because at the time this work was done the $>30 \mathrm{MeV}$ channel suffered from the temperature effect which could not be corrected.

Let us describe how we proceeded with our method. First, we define the detection threshold $N_{t h r}$ of solar neutrons as a $3 \sigma$ excess over the background. When we consider the upper limit we take into account the fluctuation of the signal plus the background. Then, our upper limit is the value which gives less than $N_{t h r}$ within the $90 \%$ C.L. of signal plus background.

Having calculated the $3 \sigma$ upper limit from our data, we estimated the number of neutrons at the Sun. First, we assumed the differential energy spectrum of neutrons at the Sun as, $f(E)=$ $C(E / 100[\mathrm{MeV}])^{\beta}$, where $C$ is the neutron flux at $100 \mathrm{MeV}$. The spectrum is modified due to the decay of neutrons in the interplanetary space, as:

$$
F(E)=\Omega P(E) C\left(\frac{E}{100[\mathrm{MeV}]}\right)^{\beta}
$$

Here, $\Omega$ is the solid angle and is given by (SNT area) $/\left(4 \pi(1[\mathrm{AU}])^{2}\right) . P(E)$ is the neutron survival probability and $\beta$ is assumed as a constant equal to -3 . Equation 5.1 is further modified by the 
neutron's attenuation in the Earth's atmosphere and the detector's response. The detection efficiency of the SNT and the NM which were already mentioned in section 2 are used. The neutron attenuation in the Earth's atmosphere was calculated based on the Shibata model[12]. We took into account incident neutrons between $70 \mathrm{MeV}$ and $1000 \mathrm{MeV}$ of kinetic energy, the zenith angle $\left(\theta_{\odot}\right)$ listed in Table 2 was also considered. We integrated Equation 5.1 from $70 \mathrm{MeV}$ to $1 \mathrm{GeV}$. Finally, we compared the results from the integration with the calculated upper limits. In Figure 3 the estimated upper limit of the neutron flux at the Sun for five SNTs and the CHNM are shown. In each plot are also added (colored squares) the six successful detections made in solar cycle 23 $[2,3]$.

\section{Conclusions}

We searched for solar neutrons during the period of maximum activity of solar cycle 24 . We used the collected data from the detectors that are part of the International Network of Solar Neutron Telescopes. Our examination included data in the period of October 2013 to October 2014, where more than $41 \mathrm{X}$-ray flares classified by GOES as greater than $>$ M5 occurred.

The first result is that, with our statistical analysis no excess equal or greater than $3 \sigma$ has been observed from our sample which consisted of $13 \mathrm{M}$-class (>M5) and 16 X-class flares. The second result is the estimation of the upper limit for solar neutrons. Our upper limits are consistent with the successful detections made in solar cycle 23.

\section{Acknowledgements}

The authors wish to thank to all the people who maintain operational the solar neutron telescopes and the Chacaltaya NM. We appreciate the kind help of Dr. L.X. González from UNAM for providing a new re-calculation of the detection efficiency of the Sierra Negra SNT.

\section{References}

[1] GOES X-ray flux. NOAA/NWS, 2015. http://www.swpc.noaa.gov/products/goes-x-ray-flux

[2] K. Watanabe et al., 2003, ApJ 592, 590; K. Watanabe et al., 2003, Proc. 28th ICRC; K. Watanabe et al., (2005) Proc. 29th ICRC; K. Watanabe et al., 2006, ApJ 636,1135.

[3] T. Sako et al., 2006, ApJ 651, L69-L72.

[4] E. L. Chupp et al., 1987, ApJ 318, 913.

[5] Y. Muraki et al., 1991, ApJ 400, L75.

[6] H. Tsuchiya et al., 2001, Nucl. Instr. and Meth. A 463, 183-193.

[7] K. Watanabe, 2005, PhD Thesis, Department of Physics, Nagoya University.

[8] J.F. Valdés-Galicia et al., 2004, Nucl. Instr. and Meth. A 535, 656-664.

[9] M. Moser, 2002, Diplomarbeit (M.Sc. Thesis), Science Faculty, Bern University.

[10] L.X. González et al., 2010, Nucl. Instr. and Meth. A 613, 263-271.

[11] J. M. Clem \& L.I. Dorman, 2000, Space Sci. Rev. 93, 335-359.

[12] S. Shibata, 1994, J. Geophys. Res. 99, 6651-6665. 